\title{
Implementation of Two Level QR Code (2LQR)
}

\author{
Pallavi Tekade ${ }^{1}$, Anub Vamadevan ${ }^{2}$, Sumit Sawant ${ }^{3}$, Tushar Tamhane ${ }^{4}$, Ganesh Khedkar $^{5}$ \\ Professor, Dept of Information Technology, Rajarshi Shahu College of Engineering, Savitribai Phule Pune University, \\ Pune, India ${ }^{1,2}$ \\ Student, Dept of Information Technology, Rajarshi Shahu College of Engineering, Savitribai Phule Pune University, \\ Pune, India ${ }^{3,4,5}$
}

\begin{abstract}
Quick response codes are machine readable optical label which was first created in the automated industry of Japan. Quick response codes are capable enough to store vital information in it by converting into various black and white patterns. QR codes have many applications especially in the field of message sharing and document authentication. We must lay our emphasis on the security and privacy of the QR code as there are chances of it getting into wrong hands. In this paper, we have proposed a technique known as 2-level QR code which consists of two layers of security provided to the QR code. The first level is known as public level and the second level is known as private level. We will be using Reed Solomon algorithm. Our 2-level design is capable enough to supply ample security and privacy as way as personal message sharing and document authentication are involved. During this paper, we've got studied numerous state of the art existing techniques of planning QR code at the side of their comparison with our projected two level QR code technique. In this paper, we've got studied numerous state of the art existing techniques of coming up with QR code in conjunction with their comparison with our planned two level QR code technique.
\end{abstract}

Keywords: QR Code; Reed Solomon, 2-level QR, Cryptography, Barcode.

\section{INTRODUCTION}

QR code was created by Denso Wave in Japan while working for car industry in 1994.QR codes are basically a twodimensional bar codes which are used day by day due to the technological advancements.These codes have a lot of uses. To give some examples, these QR codes can be utilized for putting away data, site diverting, following and following (for transportation tickets or brands), Entity distinguishing proof (traveller data, general store items), Uniform asset locator, Code instalments, Virtual stores, Website login, Message sharing and record verification.Subsequently of which the interest for $\mathrm{QR}$ code scanners has additionally expanded relatively.The prevalence of $\mathrm{QR}$ code is due to its robustness, easy to read feature, higher encoding capacity and small size.The accreditation of QR code was performed by Worldwide Organization of Standardization (ISO), and its entire particular can be found in [1]. A QR code encodes the data into binary form. Every data bit is representedby a black or a white module.The Reed-Solomon error correction code [2] is utilized for data encryption. Therefore, one of 4 error correction levels have picked during QR code generation.

The most minimal level can re-establish almost 7\% of harmed data, the most elevated amount can re-establish almost $30 \%$. As shown in Fig. 1,the QR code has a particular structure for geometrical adjustment and fast unravelling. Three position labels are utilized for QR code discovery and introduction revision. Three position tags are utilized for QR code discovery and introduction revision.One or more alignment patterns areutilized to code deformation adjustment.The module coordinates are set by timing patterns.Furthermore, the format information areas contain error correction level and mask pattern. The code version and errorcorrection bits are stored in the version information areas.The QR code era calculation comprises of data encoding utilizing Reed-Solomon blunder redress code, data division on codewords, use of veil example, situation of codewords and work designs into the QR code. The QR code popularity set of rules consists of the scanning manner, picture, binarization, geometrical correction and interpreting set of rules. The most straightforward sort of rich QR codes is the easy to understand QR code. The focus of these codes is to enhance the tasteful perspective of QR codes.It comprises of changing the hues and state of the modules, or of including a picture into the QR code. Distinctive plan QR code generators are proposed as free or paid applications.2 However, most of these generators prefer to sacrifice the possibility of error correction for attractive design. As of late, the rich QR code, which includes the essentialness without losing mistake adjustment limit, was presented in [3]. The creators proposed a novel technique for mixing a shading picture into the QR code, which adjusts the QR code source pixels so that the white (rsp. dark) module pixels are changed from white (rsp. dark) to any RGB values and whose luminance esteem is considered as white (rsp. dark) pixel by QR code binarization technique. Recently, the QR code steganography, which aims to hide a secret message into a QR code, was introduced. 
Vol. 6, Issue 4, April 2017

\section{LITERATURE SURVEY}

An in-depth writing has a look at turned into finished in the assist of the two stage QR code. In writing, numerous strategieshave been proposed to beautify the execution of the validation residences of the proposed $2 \mathrm{LQR}$ code. private messagesharing and archive validation shapes the premise of each hunt and query operation. This confirmation is because ofthe affectability of the applied examples to the print-and-sweep put together as very productive to decrease overheads. There are a few philosophies that have been conveyed for private message sharing and in a validation state of affairs. Theprecept range of middle of our exploration is primarily based over the generation ventures of the $2 \mathrm{LQR}$ code and the message extractionsteps. A noteworthy commitment of this given paper is explanatory and test displaying of the print-filter out prepare, whichshapes the idea of the proposed putting plans. a novel method for evaluating the flip skilled through the imageamid the checking method is proposed, which specifically abuses the information of the advanced half conditioningplan utilized by the printer.The QR code framework turned into concocted in 1994 by way of Denso Wave. Its primary cause for present became to music carsamid produce; it became meant to permit fast part scanning. [3] QR codes now are applied as a part of a far extrasizable placing, that consists of each commercial enterprise following applications and lodging arranged applications went for cellular smartphone clients (named transportable labelling). QR codes is probably applied to expose content to the purchaser, to feature avCard touch to the patron's device, to open a Uniform Aid Identifier (URI), or to create an e mail or immediatemessage. customers can create and print their personal particular QR codes for others to brush and use by using going to one ofspecific paid and loose $\mathrm{QR}$ code generating locations or applications. The innovation has because turned out to be astandout among the most-applied forms of two-dimensional barcode [4]. the inducement at the back of the writing audit changed intoto determine the type, degree, furthermore the substance of research and statistics this is directly reachable with regard toreport authentication. The discoveries of the writing survey are an asset for enhancing the high-quality, cost and time of thesystem protection. The discoveries of the writing audit additionally distinguish in which there is an absence of to be had andcritical statistics at the issue. A collection of writing resources had been explored furthermore inspected; the ones blanketedgifted diaries, proficient magazines, distinctive productions, on line distributions, and authoritative websites.

The unique wellsprings of writing and statistics and the amount of reports discovered recognized with the point to in additionalternate within the innovation.inside the first of all looked into paper "robust message stowing away for QR code [1]", response Code is applied as a part ofeveryday existence recently given that it has excessive limit encoding of information, harm resistance, shortdisentangling and different excellent traits. people can utilize it to transmit mystery facts without exam. within thesecond checked on paper "mystery concealing device making use of QR scanner tag [2]", QR code is applied two-dimensional(second) standardized identification as of past due with the upsides of bigger QR content material moreover blunderrectification potential. In mild of the mistake revision assets of QR code, we outlined a mystery concealing gadgetfor the QR standardized identification. The proposed plan can cover the thriller information into the duvet QR codewithout twisting the comprehensibility of QR substance. in the 0.33 surveyed paper "QR code utilising undetectablewatermarking in recurrence region [3]", commercial sports on the net and media ought to be ensured by way of increasing ofprotection, with assist of the $2 \mathrm{~d}$ Barcode with a sophisticated watermark in security subject. This paper recommends that QRCode is inserted with an imperceptible watermark by means of utilizing Discrete-Cosine-transform for a statistic stowing awaywithin the amassing thru the QR Code photo with undetectable watermark. within the fourth explored paper " $2^{\text {nd }}$ standardized identifications for verification: A protection approach [4]", check out the validation problem of properproducts on which $2 \mathrm{~d}$ scanner tags (2d-BC) have been published and we take the rival's perspective. The rival is standardto have entry to NC boisterous duplicates of a bona fide $2 \mathrm{~d}-\mathrm{BC}$.

\section{EXISTING SYSTEM}

The paper is organized as follows. present framework in view of searching for social values among P\&S un helpful examples and related examples. The capacity restriction may be eminently increased by code letters in order q or by way of increasing the finished example measure. Current framework comes about show a reclamation of private records. It likewise highlights the chance of utilising this QR code for document verification. on this work, personal stage developed through supplanting dark modules via specific floor example. For this current work makes use of wealthy graphical code. Non-public message is embedded by means of including distortion in print and sweep technique. These wealthy graphical codes increment hugeness by means of enhancing tasteful attitude of QR code. We with an introduction of QR code features and existing rich graphical codes in Section. Addition, the distortion added during the $\mathrm{P} \& \mathrm{~S}$ process will be discussed there. The proposed two level QR code as well as the proposed recognition method are presented in Section. The experimental results show the efficiency of the proposed recognition methods and analyse the capacities of the proposed code. 


\section{PROPOSED SYSTEM}

The two level QR code which is the proposed technique provides a two-level security to the QR code which is mainly focusing on message sharing and document authentication [6]. The first level is known as public level and the second level is known as private level. The public level QR code will store the information which can be shown publicly. The private level QR code will store the information which is secret and private. When this two level QR code is scanned from any standard QR scanner, only the public message will be shown from the scanner whereas the private message will be safe, secured and hidden.One important feature of the textured patterns used is their sensitivity to the P\&S process. To take advantage of this sensitivity, we use a pattern recognition method based on maximization of correlation values among the $\mathrm{P} \& \mathrm{~S}$ degraded versions and characterization patterns.

We have tried three different types of characterization patterns: mean patterns, median patterns (for the private message sharing scenario) and original patterns (for the document authentication scenario).

The mean and median characterization patterns give almost the same results of pattern detection. Therefore, either of them can be used in the private message sharing scenario. The best pattern recognition results were obtained, when the original patterns are used as characterization patterns.

The original patterns can be also used for the private message sharing scenario, but in this case the blind method for pattern detection cannot be performed. Information encoded in a QR code is always accessible to everyone, even if it is ciphered and therefore is only legible to authorized users (the difference between" see" and" understand").

It is impossible to distinguish an originally printed QR code from its copy due to their insensitivity to the Print andScan (P\&S) process.

\section{MODULE 1: INPUT MESSAGE.}

This is our first module. In this module, we will be giving the public and private messages as an input.

MODULE 2: STANDARD QR CODE GENERATION.

In this module, we will generate a standard QR code which will be created by encoding the public message. This QR code can be scanned by any standard QR code scanner. As far as standard QR is concerned, there is a pre-defined library "Zxing" which has to be directly imported. That Library has got all the predefined methods in order to create a QR code.

We just have to import a jar file known as Zxing's core.jar file from Maven repository.

Any QR code generated using the Zxing library can be easily scanned by a standard QR scanner.

MODULE 3: 2LQR CODE GENERATION.

For creating private QR code, we will be using Reed Solomon's algorithm. Reed Solomon's algorithm is also known as Golay's algorithm.

For creating the $2 \mathrm{LQR}$ code we will be performing the following two steps.

1.) Pattern generation.

2.) Replacement of black modules of the std. QR code with the generated patterns.

We will be selecting patterns from the database. We will create the patterns of all the alphanumeric characters along with the special symbols. And then we will store those patterns in a database.

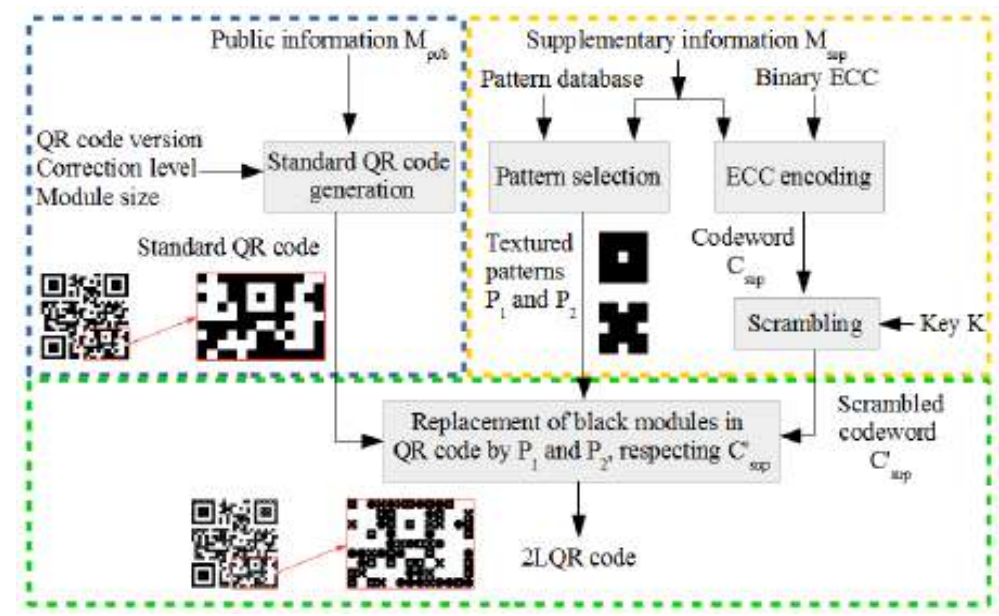


For example: Consider my message to be "A". First, we will consider the ASCII value of A.

That ASCII value is in decimal. We will convert that decimal value into binary. Afterwards, we will take a $3 \times 3$ Matrix comprising of the binary ASCII of A. The elements of the matrix will be either 1 or 0 . So, the 1 will correspond to a black spot and 0 will correspond to a white spot. Similar process will be performed on all the characters. Finally, we will get a pattern.

\section{ALGORITHMS}

1. QR code generation algorithm

2. Cryptography.

1. QR code generation

algorithm consists of information encoding using Reed-Solomon error correction code, information division on codewords, application of mask pattern, placement of codewords and function patterns. We propose to overcome these shortcomings by enriching the standard QR code encoding capacity. This enrichment is obtained by replacing its black modules by specific textured patterns. Besides the gain of storage capacity, these patterns can be designed to be sensitive to distortions due to the $\mathrm{P} \& \mathrm{~S}$ process. These patterns.

That do not introduce disruption in the standard reading process, are always perceived as black modules by any QR code reader. Therefore, even when the private information is degraded or lost in the copy, the public information is always accessible for reading.

\section{Cryptography}

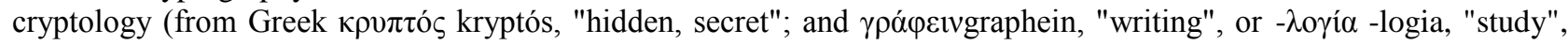
respectively) is the practice and study of techniques for secure communication in the presence of third parties called adversaries.Cryptography is closely related to the disciplines of cryptology and cryptanalysis. Cryptography includes techniques such as microdots, merging words with images, and other ways to hide information in storage or transit. However, in today's computer-centric world, cryptography is most often associated with scrambling plaintext (ordinary text, sometimes referred to as cleartext) into ciphertext (a process called encryption), then back again. Individuals who practice this field are known as cryptographers. Modern cryptography concerns itself with the following four objectives: 1) Confidentiality (the information cannot be understood by anyone for whom it was unintended) 2) Integrity (the information cannot be altered in storage or transit between sender and intended receiver without the alteration being detected) 3) Non-repudiation (the creator/sender of the information cannot deny at a later stage his or her intentions in the creation or transmission of the information).

\section{CAMPARATIVE STUDY}

\begin{tabular}{|c|c|c|c|c|c|}
\hline Code name & \multicolumn{3}{|c|}{ Storage capacity (bits/inch ${ }^{2}$ ) } & $\begin{array}{c}\text { Color } \\
\text { printing }\end{array}$ & $\begin{array}{c}\text { Copy } \\
\text { sensitivity }\end{array}$ \\
\hline HCC2D code [2] & 15048 & 0 & 15048 & Yes & No \\
\hline $\begin{array}{l}\text { Multilevel } \\
\text { 2D barcode [5] }\end{array}$ & 11224 & 0 & 11224 & No & No \\
\hline $\begin{array}{l}\text { Graphical code for } \\
\text { authentication }\end{array}$ & 0 & 0 & 0 & No & Yes \\
\hline $\begin{array}{l}\text { QR code with } \\
\text { hidden message [3] }\end{array}$ & 7548 & 3102 & 10650 & No & No \\
\hline Proposed 2LQR code $[6$ & 7548 & 6386 & 13934 & No & Yes \\
\hline
\end{tabular}

\section{CONCLUSION}

In this paper, a new wealthy code called two level QR ( $2 \mathrm{LQR})$ Code is proposed.2LQR code can be used for secure private information sharing and confirmation component. This $2 \mathrm{LQR}$ code has two levels: a public level and a private level. The public level can be read by any QR code reading software, while the private level needs a specific software with specific input information. The proposed $2 \mathrm{LQR}$ code increases the storage capacity of the classical QR code due to its supplementary reading level. Experiment results show that the storage capacity is improved by up to $28 \%$. Above calculation certainly utilizes Reed-Solomon codes for encoding mystery messages earlier than inserting the end result message into QR code. there are numerous elements of Reed-Solomon codes, the end result message probable is 
decoded via the animal constrain calculation of exact signatures from testing data images produces error in verifying the signatures. [8]

\section{REFERENCES}

[1] Information Technology-Automatic Identification and Data Capture Techniques—Bar Code Symbology-QR Code, ISO/IEC Standard 18004:2000, 2000.

[2] J. Rouillard, “Contextual QR codes,” in Proc. IEEE 3rd Int. Multi-Conf.Comput. Global Inf. Technol. (ICCGI), Jul./Aug. 2008, pp. 50-55.

[3] T. V. Bui, N. K. Vu, T. T. P. Nguyen, I. Echizen, and T. D. Nguyen, "Robust message hiding for QR code,” in Proc. IEEE 10th Int. Conf

[4] P.-Y. Lin, Y.-H. Chen, E. J.-L. Lu, and P.-J. Chen, "Secret hidingmechanism using QR barcode," in Proc. IEEE Int. Conf. SignalImageTechnol. Internet-Based Syst. (SITIS), Dec. 2013, pp. 22-25.

[5] J. Picard, "Digital authentication with copy-detection patterns," Proc.SPIE, vol. 5310, pp. 176-183, Jun. 2004.

[6] IuliiaTkachenko, William Puech, Senior Member, IEEE, Christophe Destruel, Olivier Strauss, Jean-Marc Gaudin, and Christian Guichard, "Two-Level QR Code for Private Message Sharingand Document Authentication," IEEE TRANSACTIONS ON INFORMATION FORENSICS AND SECURITY, VOL. 11, NO. 3, MARCH 2016

[7] L. Yu, X. Niu, and S. Sun," Print-and-scan model and the watermarking countermeasure,” Image Vis. Comput., vol. 23, no. 9, pp. 807-814, 2005.

[8] S. V. Voloshynovskiy, O. Koval, F. Deguillaume, and T. Pun," Visual communications with side information via distributed printing channels: Extended multimedia and security perspectives," Proc. SPIE, vol. 5306, pp. 428-445, Jun. 2004 